UCSD/PTH 98-11

\title{
Systematic Study of Theories with Quantum Modified Moduli. II
}

\author{
Benjamín Grinstein[迆 and Detlef R. Nolte[团] \\ Department of Physics, University of California at San Diego \\ La Jolla, CA 92093
}

March 1998

\begin{abstract}
We complete the process of classifying all supersymmetric theories with quantum modified moduli. We present all the supersymmetric gauge theories based on a simple orthogonal or exceptional group that exhibit a quantum modified moduli space. The quantum modified constraints of theories derived from s-confining theories are invariant under all symmetries. However, theories that cannot be obtained by a deformation of an s-confining theory may have constraints that are covariant, rather than invariant.
\end{abstract}




\section{Introduction}

There has been much recent progress in our understanding of the the phase structure of supersymmetric gauge field theories [2]. Seiberg[1] considered SUSY QCD with $N_{c}$ colors and $N_{f}$ quarks in the fundamental and antifundamental representation of $S U\left(N_{c}\right)$. For $N_{f} \leq N_{c}+1$ Seiberg found a description of the original theory in terms of the confined degrees of freedom which are gauge invariant operators in the matter fields. Using the symmetries and the holomorphy of the superpotential he determined the superpotential of these theories. These superpotentials can only be generated non-perturbatively because of the non-renormalisation theorem for the superpotential in perturbation theory. In this range of $N_{f}$ one can distinguish three types of theories based on the changes made on the classical vacuum. The superpotential for $N_{f}<N_{c}$ lifts the classical vacuum. For $N_{f}=N_{c}$ it just modifies the classical vacuum. This modification appears as a quantum change to a classical constraint between the gauge invariant degrees of freedom. For $N_{f}=N_{c}+1$ classical and quantum vacua are the same. There are still constraints between the gauge invariant operators, but they are not modified. These qualitatively different phenomena can be looked for in theories with different matter content and different gauge groups. Generally,

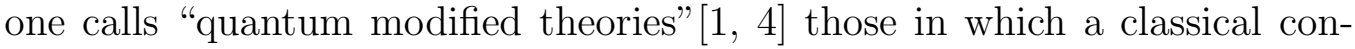
straint between the gauge invariant degrees of freedom is changed due to quantum effects. Following Csaki et al[3] we call the analogs to SUSY QCD $N_{f}=N_{c}+1$ "s-confining" theories. Each type of theory needs a specific superpotential to implement such a vacuum modification. The special features of the superpotentials are used to classify the s-confining theories [3] and the quantum modified theories [4].

In this paper we complete our work on the classification of quantum modified theories [4]. We do this in two different ways. First we classify quantum modified theories for the simple gauge groups not considered in Ref. [4. That means we concentrate on the $\mathrm{SO}$ and exceptional $\left(G_{2}, F_{4}, E_{6,7,8}\right)$ groups. We give a detailed description of the quantum modified theories which are not derived from s-confining theories. Second we elaborate on a claim in [4. We argued there that in theories with a covariantly modified moduli space (cQMM theories) new branches can appear on certain points in moduli space. At the branch the Lagrange multiplier is massless and it is therefore responsible for the branch. The identity of the Lagrange multiplier was not known 
and therefore the new branch seemed to be mysterious. We study a theory based on a non-simple gauge group in which the c-QMM theory is derived from a s-confining theory. It is now possible to identify the Lagrange multiplier with a composite field of the parent s-confining theory. By moving through the moduli space the mass of this composite field changes. At certain points on moduli space the mass of this particle vanishes and there the supersymmetric vacuum has a new branch. For the c-QMM theories based on a simple gauge group such an interpretation of the Lagrange multiplier is difficult to proof because they cannot be derived from s-confining theories.

We have found that if a simple group gauge theory derived from an sconfining theory has a quantum modified moduli, then this moduli is described by a quantum modified constraint that is invariant under all symmetries. For these "i-QMM theories" the Lagrange multipliers are again composite fields of the parent s-confining theories. If there is just one constraint then there cannot be a new branch on which the Lagrange multiplier becomes massless. SUSY-QCD is an example of such a theory. But if the theory has multiple constraints new branches can occur. We will give an example in which this happens.

In section 2 we collect the tools needed for a systematic classification of all quantum modified theories (for details and derivations see [4]). In this section we also list all the flows. A discussion of new branches found in c-QMM theories or theories with multiple constraints can be found in section 3. Our results for the $\mathrm{SO}$ and exceptional groups are presented in section 4 . Section 5 has conclusions. In an appendix we present a method which helps in determining how theories flow. We used this method to find the branches for the exceptional groups.

\section{Index Condition and Flows}

\subsection{The Index Condition}

Quantum modified theories have at least one constraint between the gauge invariant operators $\phi_{i}$. At the classical level, the constraints are schematically of the form

$$
\sum_{n=1}^{m}\left(\prod_{i=1}^{k_{n}} \phi_{i}\right)_{n}=0
$$


Quantum dynamics modifies this constraint to

$$
\sum_{n=1}^{m}\left(\prod_{i=1}^{k_{n}} \phi_{i}\right)_{n}=\prod_{i} \phi_{i} \Lambda^{p} .
$$

The important feature is the scale $\Lambda$ appearing on the right side of Eq. (酉). If the original classical constraint is not invariant under all symmetries, one has to multiply the $\Lambda^{p}$ term by some gauge invariant operators which produces a constraint covariant under all the symmetries. Generally the symmetries do not fix the gauge invariant operator uniquely. By flowing the covariant theories to known theories one can determine the gauge invariant operator uniquely. We refer to these covariant theories as c-QMM theories and for theories with invariant classical constraints as i-QMM theories. This distinction is physically important. We describe this in Ref. [4] and in section 3 .

To understand the index constraint we assign a $U(1)_{R}$ symmetry to each matter field separately and require that both sides have the same charge under all the $U(1)_{R}$ symmetries. Because the scale $\Lambda$ appears one one side of the constraint there is at least one field which appears as a higher power on the left than on the right. Therefore the $U(1)_{R}$ charge of this field has to be zero. Otherwise the constraint would not be covariant under this $U(1)_{R}$ symmetry. But gauge anomaly cancellation for this $U(1)_{R}$ symmetry requires:

$$
\sum_{i=1}^{n} \mu_{i}-\mu_{G}=0
$$

We call this the index condition. For another argument and more details see Refs. [3, 4].

\subsection{The Flow}

\subsubsection{The Flow of the Theories}

The index condition is only a necessary condition for a quantum deformation of the moduli. To find out if a candidate theory actually has a QMM, one must make further investigations. As the next step to sort out all QMM theories we consider points in the classical moduli space where the gauge

group of the candidate theory is broken. The gauge fields which correspond to the broken generators acquire a mass proportional to the vacuum expectation 
value of the Higgs field. These massive gauge superfields pair up with chiral superfields which become massive through the super-Higgs mechanism as well. Together they form a massive supermultiplet. We integrate out these heavy degrees of freedom. The new theory, which is an effective theory of the original 'UV' theory, should be in a phase consistent with the UV theory being in a quantum modified phase. We refer to this as 'the flow' of the UV theory to an effective theory. If the theory flows to a theory in a Coulomb phase [5] we say that the theory has a Coulomb branch, not a QMM. By studying the flow we can, therefore, rule out quite a few theories which fulfill the index condition.

It is useful to tabulate the manner in which theories may flow. Below we list the gauge groups together with their particle content. For a $S O(2 N)$ theory with $s$ spinors, $s^{\prime}$ conjugate spinors and $q$ vector representations as the matter contents we write $\left(s, s^{\prime}, q\right)$. For a $S O(2 N+1)$ theory with $s$ spinors and $q$ vector representations as the matter contents we write $(s, q)$. For other theories the matter is presented in square brackets and is represented by the Young tableaux of the corresponding representation, with a possible multiplier when there are more than one field for that representation. We do not list any gauge singlets that may remain in the effective theory. These are not all the possible flow diagrams. They were, however, sufficient for our classification work.

$$
\begin{gathered}
\text { Simple Gauge Group [adjoint] } \longrightarrow \text { Coulomb branch } \\
\qquad O(N)[(N-2) \square] \longrightarrow \text { Coulomb branch }
\end{gathered}
$$



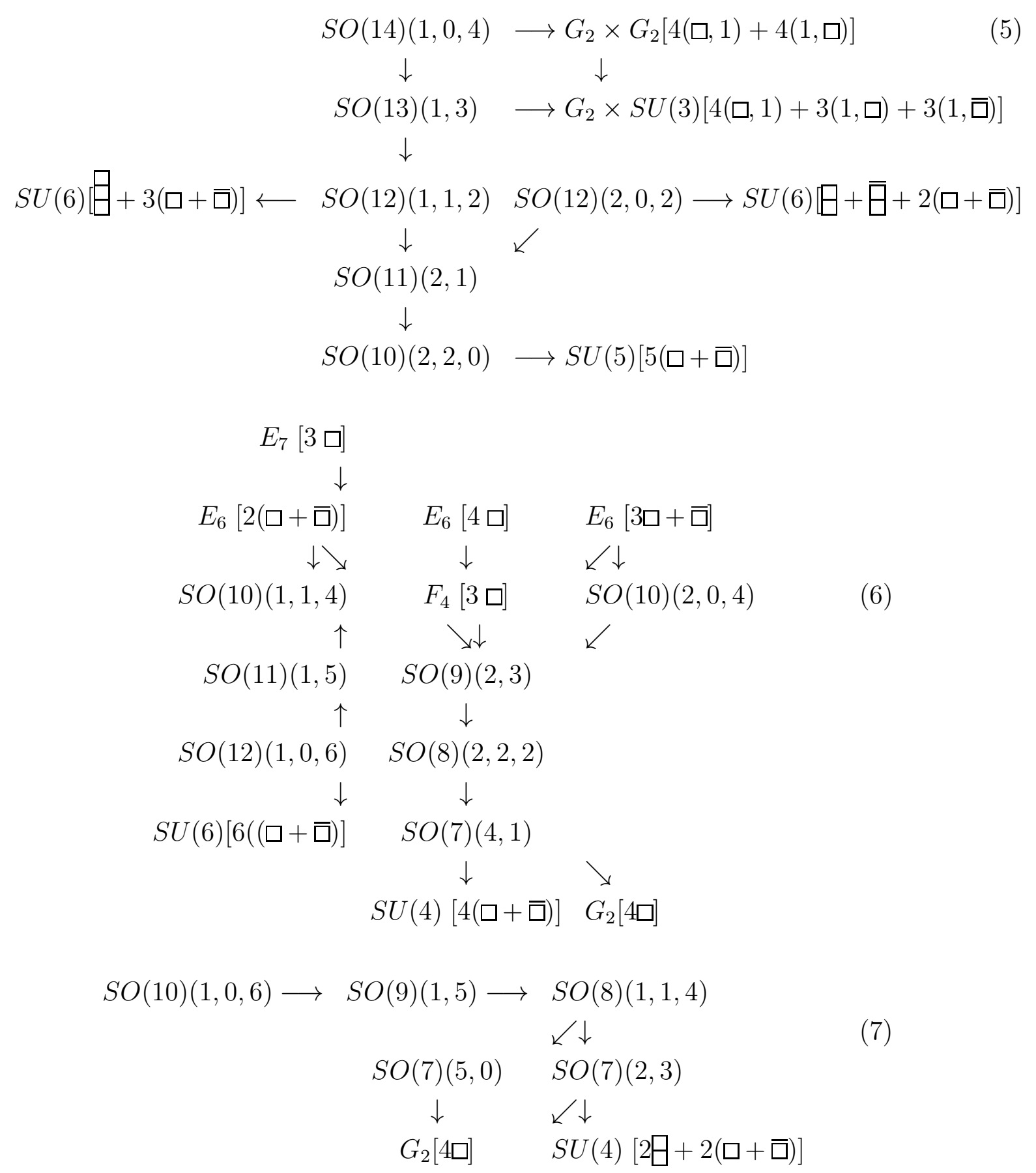


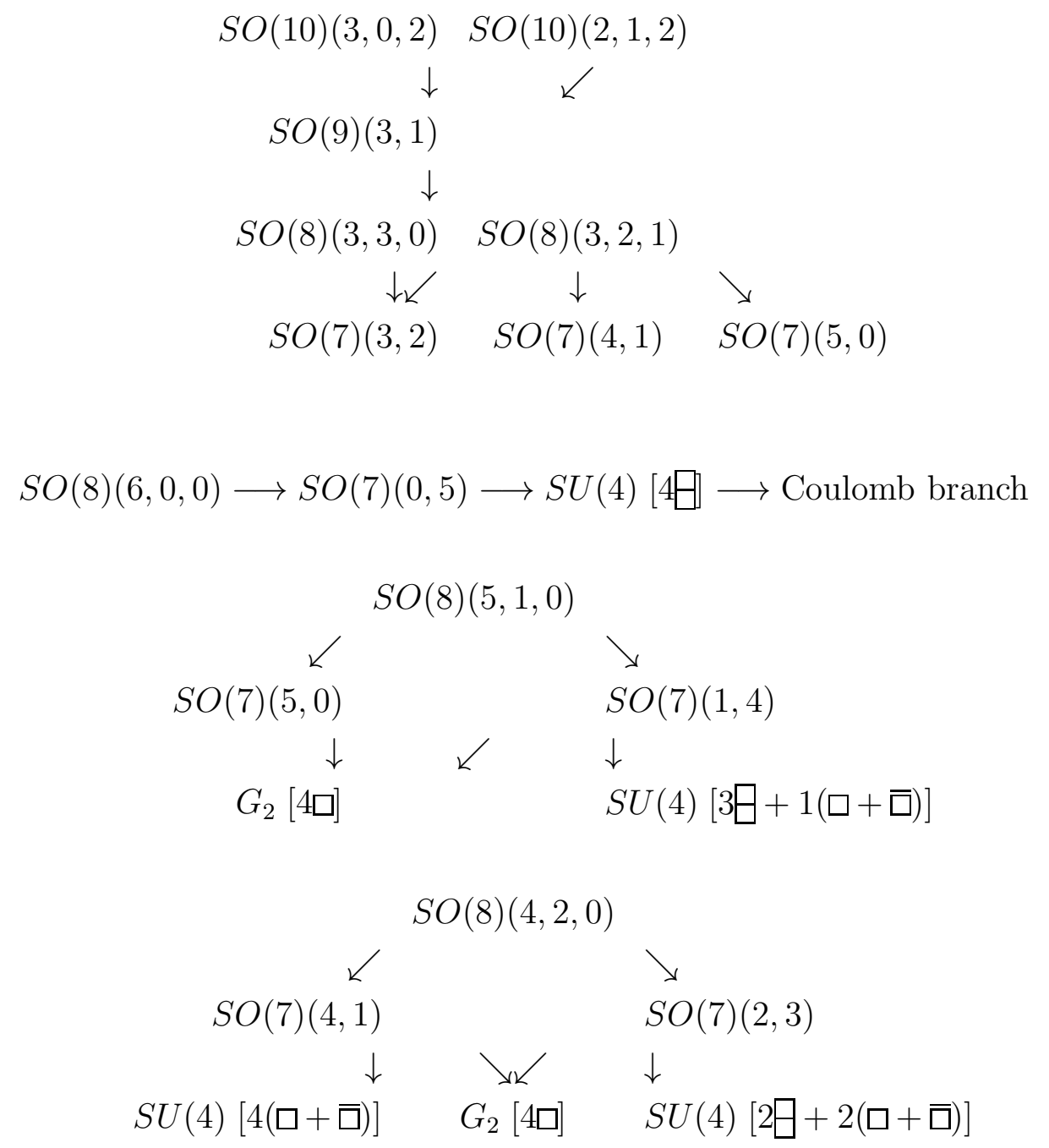

\section{New Branches}

\subsection{Branches in c-QMM Theories}

In our previous publication we observed that c-QMM theories may have a new branch on which the Lagrange multiplier becomes massless. This happens when some of the composites have infinite expectation values. Such a scenario raises some obvious questions. How can there be a new massless field if some of the composites have infinite expectation value? Infinite expectation value 
usually means that the theory is weakly coupled. But at weak coupling one does not expect to get new massless fields. The resolution to this puzzle is that the branch actually is not at weak coupling and therefore new massless fields are not impossible. The situation is similar to that in the SeibergWitten model where new massless fields at strong coupling are associated with a new branch [6, 7]. Another issue is the physical identification of the Lagrange multiplier. We argue it is a massive gauge invariant field away from the new branch at infinity.

To answer these questions we look at the simple example $S U(2) \times S U(2)$ with matter in the $(2,2)+4(2,1)+2(1,2)$ representation, which is an sconfining theory 10. By adding a mass to two $(2,1)$ fields and integrating these out one reaches a c-QMM theory, namely $S U(2) \times S U(2)$ with $(2,2)+2(2,1)+2(1,2)$ matter [11]. In this example we are able to identify the Lagrange multipliers with heavy mesons of the parent s-confining theory. Thus a meson made out of heavy quarks becomes massless at the new branch, an interesting and puzzling phenomenon; in this connection, see [12].

To be specific denote by $q_{\alpha \dot{\alpha}}, l_{\alpha i}$ and $r_{\dot{\alpha} J}$ the matter fields transforming respectively in the $(2,2),(2,1)$ and $(1,2)$ representations of $S U(2) \times S U(2)$. Here $i=1, \ldots, 4$ and $J=1,2$ are flavor indices, and $\alpha$ and $\dot{\alpha}$ are gauge indices. The local gauge invariant operators (mesons) are:

$$
\begin{gathered}
M_{i J}=q_{\alpha \dot{\alpha}} l_{\beta i} r_{\dot{\beta} J} \epsilon^{\alpha \beta} \epsilon^{\dot{\alpha} \dot{\beta}}, X=q_{\alpha \dot{\alpha}} q_{\beta \dot{\beta}} \epsilon^{\alpha \beta} \epsilon^{\dot{\alpha} \dot{\beta}}, L_{i j}=l_{\alpha i} l_{\beta j} \epsilon^{\alpha \beta}, R=r_{\dot{\alpha} 1} r_{\dot{\beta} 2} \epsilon^{\dot{\alpha} \dot{\beta}}, \\
Y=r_{\dot{\alpha} 1} r_{\dot{\beta} 2} q_{\alpha \dot{\gamma}} q_{\beta \dot{\eta}} \epsilon^{\alpha \beta} \epsilon^{\dot{\alpha} \dot{\gamma}} \epsilon^{\dot{\beta} \dot{\eta}} \text { and } W_{i j}=l_{\alpha i} l_{\beta j} q_{\gamma \dot{\alpha}} q_{\eta \dot{\beta}} \epsilon^{\alpha \gamma} \epsilon^{\beta \eta} \epsilon^{\dot{\alpha} \dot{\beta}}
\end{gathered}
$$

This theory is s-confining and if the two $S U(2)$ factors have the same coupling scale $\Lambda$ the effective superpotential is

$$
W=-\frac{X \operatorname{Pf} L-\frac{1}{4} W \cdot L}{\Lambda^{3}}-\frac{R \operatorname{Pf} W-\frac{1}{2} M^{2} \cdot W}{\Lambda^{7}}
$$

where $W \cdot L=W_{i j} L_{k l} \epsilon^{i j k l}$ and $M^{2} \cdot W=M_{i 1} M_{j 2} W_{k l} \epsilon^{i j k l}$. One may now deform this theory by adding a mass term making two of the $l_{i}$ fields heavy, obtaining at low energies $S U(2) \times S U(2)$ with $(2,2)+2(2,1)+2(1,2)$, a theory with a c-QMM. From the superpotential

$$
W=-\frac{X \operatorname{Pf} L-\frac{1}{4} W \cdot L}{\Lambda^{3}}-\frac{R \operatorname{Pf} W-\frac{1}{2} M^{2} \cdot W}{\Lambda^{7}}+m L_{34}
$$


one easily finds that at any point in the moduli space the fields $M_{i J}, L_{i j}$ and $W_{i j}$, with $i$ or $j=3$ or 4 are heavy and can be integrated out. However, special care must be exercised handling the fields $L_{34}$ and $W_{34}$. Leaving these in, the resulting superpotential is

$$
W=\frac{L_{34}}{\Lambda^{3}}\left[X L_{12}+W_{12}+m \Lambda^{3}\right]+\frac{W_{34}}{\Lambda^{7}}\left[-\Lambda^{4} L_{12}+R W_{12}-M^{2}\right]
$$

where $M^{2}=M_{11} M_{22}-M_{12} M_{21}$. We see that at generic points $L_{34}$ and $W_{34}$ get massive by pairing up with linear combination of fields, but the particular combination of fields that remains light depends on the specific point in the moduli. This is why it is appropriate to retain the heavy fields $L_{34}$ and $W_{34}$ in the low energy description.

Now, the superpotential of Eq. (14) is appropriate for the $S U(2) \times S U(2)$ theory with $(2,2)+2(2,1)+2(1,2)$. In the context of this theory the fields $L_{34}$ and $W_{34}$ are Lagrange multipliers enforcing two quantum modified constraints. A more familiar form of the superpotential is found by inserting the $L_{34}$ constraint into the $W_{34}$ constraint, obtaining

$$
W=\frac{W_{34}}{\Lambda^{7}}\left[R X L+\operatorname{Pf} \hat{M}+R \Lambda^{4}+L \Lambda^{4}\right],
$$

where $L=L_{12}$ and $\hat{M}_{i J}=M_{i J}$ for $i, J=1,2$. We see that the Lagrange multiplier of the standard description is $W_{34} / \Lambda^{7}$, a meson of the underlying s-confining theory.

Although one should retain both Lagrange multipliers as in Eq. (14) to describe the whole moduli space, the partially integrated form of Eq. (15) is sufficient to identify the new branches at infinity. One only needs to set $X=\epsilon^{-1}, R=L=-\epsilon \Lambda^{4}$ as $\epsilon \rightarrow 0$. On this trajectory $W_{34}$ is massive and the equations of motion are satisfied only if $W_{34}=0$. But in the limit the equations are satisfied automatically, for any $W_{34}$. On this branch, $W_{34}$ is a massless field with massive constituents.

At the new branch, the gauge symmetry is broken, $S U(2) \times S U(2) \rightarrow$ $S U(2)$. The unbroken $S U(2)$ is strongly coupled, even though the symmetry breaking occurs at infinity on the moduli space. In fact, as we approach the branch on the hypersurface $W_{34}=0$ the gauge symmetry is completely broken, but with $S U(2) \times S U(2) \rightarrow S U(2)$ at the high scale $X=\epsilon^{-1}$ while the diagonal $S U(2)$ is broken only at the low scale $R=L=-\epsilon \Lambda^{4}$. 
We emphasize that we cannot proof the existence of this new branch and its physical consequences. Since at the branch the theory is strongly coupled we have no means of computing the Kahler potential, and therefore we cannot discount the possibility that it is singular at the new branch.

\subsection{Branches for Theories with Multiple Constraints}

For QMM-theories derived from s-confining theories the number of constraints between the composites may be greater than one. One of the constraints must be quantum modified. This quantum modified constraint arises from integrating out the composite corresponding to the tree level mass term added. For SUSY QCD the mass term is $W_{\text {mass }}=m\left(Q_{N_{f}} \bar{Q}_{N_{f}}\right)$ and the corresponding composite is the meson type operator $M_{N_{f} N_{f}}$. Integrating this out gives the quantum modified constraint. Generally there can be other constraints. The number of constraints is given by the formula 四

$$
N_{\text {Con }}=N_{\text {Ops }}-\left(N_{\text {Fund }}-\operatorname{dim} G\right)
$$

where $N_{\text {Fund }}, N_{\text {Ops }}$ and $N_{\text {Con }}$ are the number of fundamental chiral superfields, the number of independent gauge invariant operators and the number of constraints, respectively, and $\operatorname{dim} G$ is the dimension of the gauge group. The additional constraints can be derived by integrating out other massive composite fields from the parent s-confining theory. Because there is no tree level mass term introduced for these fields they generally generate constraints without quantum modifications. The Lagrange multipliers correspond to these massive composite fields. It is easy to see that these theories can have new branches on which the Lagrange multipliers are massless.

As an example we consider $S U(4)$ with $[2 \boxminus+2(\square+\square)]$ which was considered in [3]. The matter contents and the gauge invariant operators are summarized in the next table: 


\begin{tabular}{|l|c|ccc|c|}
\hline & $S U(4)$ & $S U(2)$ & $S U(2)$ & $S U(2)$ & d.o.f. \\
\hline$A$ & $\boxminus$ & $\square$ & 1 & 1 & $2 \times 6$ \\
$Q$ & $\square$ & 1 & $\square$ & 1 & $2 \times 4$ \\
$\bar{Q}$ & $\square$ & 1 & 1 & $\square$ & $2 \times 4$ \\
\hline$M_{0}=(Q \bar{Q})$ & 1 & 1 & $\square$ & $\square$ & 4 \\
$M_{2}=(Q A A \bar{Q})$ & 1 & 1 & $\square$ & $\square$ & 4 \\
$T=(A A)$ & 1 & $\square$ & 1 & 1 & 3 \\
$H=(A Q Q)$ & 1 & $\square$ & 1 & 1 & 2 \\
$H=(A \bar{Q} \bar{Q})$ & 1 & $\square$ & 1 & 1 & 2 \\
$\lambda$ & 1 & 1 & 1 & 1 & 0 \\
$\mu$ & 1 & 1 & 1 & 1 & 0 \\
\hline
\end{tabular}

The number of constraints adds up to 2 and the superpotential is

$$
W=\lambda\left[T^{2} M_{0}^{2}-T H \bar{H}-M_{2}^{2}-\Lambda^{8}\right]+\mu\left[M_{0} M_{2}+H \bar{H}\right] .
$$

There is a new branch with a massless $\mu$. For

$$
M_{0} \sim \epsilon^{p_{1}}, M_{2} \sim \epsilon^{p_{2}}, H \sim \epsilon^{p_{3}}, \bar{H} \sim \epsilon^{p_{4}} \text { and } T \sim \epsilon^{-p_{1}},
$$

$\mu$ is arbitrary in the limit $\epsilon=0$. Here we assume that all the $p_{i}$ are positive and $p_{3}+p_{4} \geq p_{1}$.

One can see that this is quite generic. For example, if the $\mu$-constraint has no linear term and there is a field in the $\lambda$-constraint which is not in the $\mu$-constraint the $\mu$ branch is guaranteed. This new branch is reached if the $\mathrm{T}$ fields have infinite field strength. This resembles the in c-QMM theories. But for QMM-theories with multiple constraints this does not have to be generic. One can easily imagine how one could get a new branch at finite field strength. For example, a superpotential of the schematic form

$$
W=\lambda\left[A B+C D-\Lambda^{p}\right]+\mu\left[A C E-D^{2}\right]
$$

has a new branch at $\epsilon \rightarrow 0$ with

$$
C \sim \epsilon^{p_{1}}, D \sim \epsilon^{p_{2}}, E \sim \epsilon^{p_{3}} \text { and } p_{i}>0
$$

if $(A B) \sim \Lambda^{p}$, with no composite field of infinite field strength. 


\section{The Quantum Modified Theories}

\section{1 $S O(N)$ Theories}

In this section we list all the theories fulfilling the index constraint. For each theory we list also if the theory is C-QMM and the number of constraints. The consistency of these tables can be checked in different ways. A useful condition follows from the observation that i-QMM theories can never flow into c-QMM theories. Another, perhaps obvious, fact is that the number of constraints can never increase while flowing from one theory down to another.

Most of the quantum modified theories for SO gauge groups can be derived from s-confining theories. This is easily accomplished by using the tab-

ulated s-confining theories[3]. The gauge invariant operators are the same if a flavor is left out. The superpotential can be generated by integrating out one flavor from the s-confining superpotentials.

All the other quantum modified theories, those not derived from s-confining theories, are already in the literature. The $S O(10)(1,0,6)$ theory was first derived by Pouliot and Strassler[9]. The results for $S O(9)(1,5), S O(8)(1,1,4)$ and $S O(7)(2,3)$ are easily generated by considering $S O(10)(1,0,6)$ along some flat directions 13. The c-QMM theory $S O(8)(0,1,5)$ was also derived by Pouliot and Strassler 9] from which $S O(7)(1,4)$ follows along flat directions 13. The results are summarized in Table 1 . 


\begin{tabular}{|l|l|l|l|}
\hline$S O(N)$ & $(0,0, N-2)$ & Coulomb branch & \\
$S O(N)$ & adjoint & Coulomb branch & \\
$S O(14)$ & $(1,0,4)$ & i-quantum modified from s-confining theory & 2 \\
$S O(13)$ & $(1,3)$ & i-quantum modified from s-confining theory & 2 \\
$S O(12)$ & $(1,0,6)$ & i-quantum modified from s-confining theory & 1 \\
$S O(12)$ & $(2,0,2)$ & i-quantum modified from s-confining theory & 3 \\
$S O(12)$ & $(1,1,2)$ & i-quantum modified from s-confining theory & 2 \\
$S O(11)$ & $(1,5)$ & i-quantum modified from s-confining theory & 1 \\
$S O(11)$ & $(2,1)$ & i-quantum modified from s-confining theory & 2 \\
$S O(10)$ & $(4,0,0)$ & i-quantum modified from s-confining theory & 1 \\
$S O(10)$ & $(3,0,2)$ & i-quantum modified from s-confining theory & 1 \\
$S O(10)$ & $(2,0,4)$ & i-quantum modified from s-confining theory & 1 \\
$S O(10)$ & $(3,1,0)$ & i-quantum modified from s-confining theory & 2 \\
$S O(10)$ & $(2,1,2)$ & i-quantum modified from s-confining theory & 1 \\
$S O(10)$ & $(1,1,4)$ & i-quantum modified from s-confining theory & 1 \\
$S O(10)$ & $(2,2,0)$ & i-quantum modified from s-confining theory & 2 \\
$S O(10)$ & $(1,0,6)$ & i-quantum modified & 1 \\
$S O(9)$ & $(3,1)$ & i-quantum modified from s-confining theory & 1 \\
$S O(9)$ & $(2,3)$ & i-quantum modified from s-confining theory & 1 \\
$S O(9)$ & $(1,5)$ & i-quantum modified & 1 \\
$S O(8)$ & $(5,1,0)$ & c-quantum modified \\
$S O(8)$ & $(4,2,0)$ & i-quantum modified from s-confining theory & 2 \\
$S O(8)$ & $(3,3,0)$ & i-quantum modified from s-confining theory & 1 \\
$S O(8)$ & $(4,1,1)$ & i-quantum modified & 1 \\
$S O(8)$ & $(3,2,1)$ & i-quantum modified from s-confining theory & 1 \\
$S O(8)$ & $(2,2,2)$ & i-quantum modified from s-confining theory & 1 \\
$S O(7)$ & $(5,0)$ & i-quantum modified from s-confining theory & 1 \\
$S O(7)$ & $(4,1)$ & i-quantum modified from s-confining theory & 1 \\
$S O(7)$ & $(3,2)$ & i-quantum modified from s-confining theory & 1 \\
$S O(7)$ & $(2,3)$ & i-quantum modified \\
$S O(7)$ & $(1,4)$ & c-quantum modified & 1 \\
\hline
\end{tabular}

Table 1: These are all $S O$ theories satisfying $\sum_{j} \mu_{j}-\mu_{G}=0$. We list the gauge group and the field content of the theories in the first and second column. For a $S O(2 N)$ theory with $s$ spinors, $s^{\prime}$ conjugate spinors and $q$ vector representations as the matter contents we write $\left(s, s^{\prime}, q\right)$. For a $S O(2 N+1)$ theory with $s$ spinors and $q$ vector representations as the matter contents we write $(s, q)$. In the third column, we indicate whether the theory has a quantum modified moduli space or a Coulomb branch. The prefix "i" indicates an invariant quantum modification and the prefix "c" a covariant quantum modification. For invariant quantum modified theories we indicate if the quantum modified theory can be derived from a s-confining theory.In the last column we indicate the number of constraints. 


\subsection{Theories with Exceptional Groups}

In the following we describe all theories based on exceptional groups which have a quantum modified moduli. To be complete we list all such theories, even though some of them have already been introduced in the literature [14]. For each theory we give a table listing, in the first column, the matter content and all the local gauge invariant operators. The second column lists the transformation properties under the gauge symmetry group (non-trivial only for the matter content) and in the remaining columns we give the transformation properties under the global "flavor" symmetry groups. Lagrange multipliers are fields and as such we include them in our lists.

As opposed to what is done in Ref. [4] we do not write down the explicit contraction of all the indices for the gauge invariant operators. Detailed analysis like that of Ref. [4] is necessary in order to write down the exact form of the classical constraints, which can be checked explicitly. This was useful for some involved cases there, but the behavior of the theories in this paper can be determined without resorting to such detailed analysis. While we cannot write down the superpotentials, we give under each table the degree of homogeneity of the superpotential with respect to each matter field.

To find the schematic form of a superpotential one simply forms all the flavor and gauge invariant operators out of the listed gauge invariant operators. The terms appearing in the superpotential are only constrained by the fixed power under which each fundamental field must appear (which, as

stated above, is listed under each table). We derived these by flowing down to known theories. We checked 't Hooft anomaly matching for all the theories.

Table 2 summarizes the findings for exceptional groups. 


\begin{tabular}{|l|l|l|l|}
\hline$E_{8}$ & $\square=$ adjoint & Coulomb branch & \\
$E_{7}$ & adjoint & Coulomb branch & \\
$E_{7}$ & $3 \square$ & i-quantum modified & 1 \\
$E_{6}$ & adjoint & Coulomb branch & \\
$E_{6}$ & $4 \square$ & i-quantum modified & 1 \\
$E_{6}$ & $3 \square+\bar{\square}$ & i-quantum modified & 1 \\
$E_{6}$ & $2(\square+\bar{\square})$ & i-quantum modified & 1 \\
$F_{4}$ & adjoint & Coulomb branch & \\
$F_{4}$ & $3 \square$ & i-quantum modified & 1 \\
$G_{2}$ & $4 \square$ & i-quantum modified from s-confining & 1 \\
$G_{2}$ & adjoint & Coulomb branch & \\
\hline
\end{tabular}

Table 2: These are all exceptional theories satisfying $\sum_{j} \mu_{j}-\mu_{G}=0$. We list the gauge group and the field content of the theories in the first and second column. In the third column, we indicate whether the theory has a quantum modified moduli space or a Coulomb branch. The prefix "i" indicates an invariant quantum modification and the prefix "c" indicates a covariant quantum modification. Only one of the theories can be derived from s-confining theories.In the last column we indicate the number of constraints.

\subsection{1 $\quad F_{4}$ with $3 \square$}

\begin{tabular}{|l|c|cc|}
\hline & $F_{4}$ & $S U(3)$ & $U(1)_{R}$ \\
\hline$Q$ & $\square$ & $\square$ & 0 \\
\hline$Q^{2}$ & 1 & $\square$ & 0 \\
$Q^{3}$ & 1 & $\square$ & 0 \\
$Q^{4}$ & 1 & $\boxplus$ & 0 \\
$Q^{5}$ & 1 & $\bar{\square}$ & 0 \\
$Q^{6}$ & 1 & 1 & 0 \\
$Q^{9}$ & 1 & 1 & 0 \\
$\lambda$ & 1 & 1 & 2 \\
\hline
\end{tabular}

$Q$ appears as $Q^{18}$ in the superpotential. 
4.2.2 $E_{6}$ with $2(\square+\bar{\square})$

\begin{tabular}{|l|c|ccc|}
\hline & $E_{6}$ & $S U(2)$ & $S U(2)$ & $U(1)_{R}$ \\
\hline$Q$ & $\square$ & $\square$ & 1 & 0 \\
$\bar{Q}$ & $\square$ & 1 & $\square$ & 0 \\
\hline$Q \bar{Q}$ & 1 & $\square$ & $\square$ & 0 \\
$Q^{3}$ & 1 & $\square$ & 1 & 0 \\
$\bar{Q}^{3}$ & 1 & 1 & $\square$ & 0 \\
$Q^{2} \bar{Q}^{2}$ & 1 & $\square$ & $\square$ & 0 \\
$Q \bar{Q}^{4}$ & 1 & $\square$ & 1 & 0 \\
$Q^{4} \bar{Q}$ & 1 & 1 & $\square$ & 0 \\
$Q^{3} \bar{Q}^{3}$ & 1 & $\square$ & $\square$ & 0 \\
$Q^{4} \bar{Q}^{4}$ & 1 & 1 & 1 & 0 \\
$Q^{6} \bar{Q}^{6}$ & 1 & 1 & 1 & 0 \\
$\lambda$ & 1 & 1 & 1 & 2 \\
\hline
\end{tabular}

$Q$ appears as $Q^{12}$ in the superpotential and $\bar{Q}$ as $\bar{Q}^{12}$.

\subsection{3 $\quad E_{6}$ with $3 \square+\bar{\square}$}

\begin{tabular}{|l|c|ccc|}
\hline & $E_{6}$ & $S U(3)$ & $U(1)_{A}$ & $U(1)_{R}$ \\
\hline$Q$ & $\square$ & $\square$ & 1 & 0 \\
$\bar{Q}$ & $\bar{\square}$ & 1 & -3 & 0 \\
\hline$Q \bar{Q}$ & 1 & $\square$ & -2 & 0 \\
$Q^{3}$ & 1 & $\square$ & 3 & 0 \\
$\bar{Q}^{3}$ & 1 & 1 & -9 & 0 \\
$Q^{2} \bar{Q}^{2}$ & 1 & $\square$ & -4 & 0 \\
$Q^{4} \bar{Q}$ & 1 & $\boxplus$ & 1 & 0 \\
$Q^{6}$ & 1 & 1 & 1 & 0 \\
$Q^{5} \bar{Q}^{2}$ & 1 & $\boxplus$ & -1 & 0 \\
$Q^{9} \bar{Q}^{3}$ & 1 & 1 & 0 & 0 \\
$\lambda$ & 1 & 1 & 0 & 2 \\
\hline
\end{tabular}

$Q$ appears as $Q^{18}$ in the superpotential and $\bar{Q}$ as $\bar{Q}^{6}$. 


\subsection{4 $\quad E_{6}$ with $4 \square$}

\begin{tabular}{|l|c|cc|}
\hline & $E_{6}$ & $S U(4)$ & $U(1)_{R}$ \\
\hline$Q$ & $\square$ & $\square$ & 0 \\
\hline$Q^{3}$ & 1 & $\square$ & 0 \\
$Q^{6}$ & 1 & $\boxplus$ & 0 \\
$Q^{12}$ & 1 & 1 & 0 \\
$\lambda$ & 1 & 1 & 2 \\
\hline
\end{tabular}

$Q$ appears as $Q^{24}$ in the superpotential.

\subsection{5 $\quad E_{7}$ with $3 \square$}

\begin{tabular}{|l|c|cc|}
\hline & $E_{7}$ & $S U(3)$ & $U(1)_{R}$ \\
\hline$Q$ & $\square$ & $\square$ & 0 \\
\hline$Q^{2}$ & 1 & $\boxminus$ & 0 \\
$Q^{4}$ & 1 & $\square$ & 0 \\
$Q^{6}$ & 1 & $\boxplus$ & 0 \\
$Q^{8}$ & 1 & $\square$ & 0 \\
$Q^{12}$ & 1 & 1 & 0 \\
$Q^{18}$ & 1 & 1 & 0 \\
$\lambda$ & 1 & 1 & 2 \\
\hline
\end{tabular}

$Q$ appears as $Q^{36}$ in the superpotential.

\section{Conclusions}

Our results are summarized in Tables 1 and 2 . They present all SUSY field theories based on a simple orthogonal or exceptional gauge group that satisfy the index constraint $\sum \mu_{i}-\mu_{G}=0$. These theories may either have a quantum modified moduli (QMM) space or a Coulomb branch with massless photons, and the right alternative is listed in the tables. For theories with a QMM we give the finer distinction of whether the constraint that is quantum modified is invariant (i-QMM) or only covariant (c-QMM) under the global symmetries of the theory. Finally, the tables list the number of constraints needed to specify the QMM. 
This work completes the classification begun in Ref. [4], which dealt with unitary and symplectic simple gauge groups. In that work it was noted that every i-QMM theory flowed from an s-confining theory and it was shown that every s-confining theory flows to a theory with an i-QMM or a Coulomb branch. However, we have seen in this paper that there exist theories (exactly four) with an i-QMM which however cannot be obtained by flowing from sconfining theories.

Our work complements recent efforts [5, 4, 17] to understand the behavior of all SUSY gauge theories based on a simple gauge group with matter satisfying $\sum \mu_{i}-\mu_{G} \leq 0$.

As in Ref. [4] we note that theories with a c-QMM exhibit a peculiar new branch at a point on the boundary of the moduli space. Here we have elucidated some of the physics of this branch by considering this theory as a deformation of an s-confining theory. The Lagrange multiplier that becomes massless on the branch corresponds to a composite of heavy constituents! This is possible because the theory remains strongly coupled on this branch.

\section{Acknowledgments}

We are grateful to Ken Intriligator, Erich Poppitz and Witold Skiba for many helpful discussions, particularly with regard to the $S U(2) \times S U(2)$ theories. This work is supported by the Department of Energy under contract DOEFG03-97ER40506. 


\section{A Appendix}

\section{A.1 Flow of the Exceptional Groups}

In order to determine whether a theory has a QMM one must explore the whole moduli space. It is possible that a theory satisfying the index constraint $\sum_{i=1}^{n} \mu_{i}-\mu_{G}=0$ has a Coulomb branch or a branch with $\sum_{i=1}^{n} \mu_{i}-$ $\mu_{G} \neq 0$. The whole moduli space must be explored because the pattern of symmetry breaking generally dictates into which different branches the theory flows.

Finding the full moduli space of the exceptional groups, however, is difficult. Given the matter content it is useful to have at hand all possible patterns of symmetry breaking. In this appendix we describe a simple, general method for determining all possible patterns of symmetry breaking given a gauge group and matter content.

It will be useful to have an illustrative example at hand. Consider an $S U(3)$ theory with matter $A$ in the adjoint representation. There are two patterns of symmetry breaking possible, given by the expectation values

$$
\langle A\rangle=\left(\begin{array}{ccc}
1 & 0 & 0 \\
0 & 1 & 0 \\
0 & 0 & -2
\end{array}\right)
$$

or

$$
\langle A\rangle=\left(\begin{array}{ccc}
1 & 0 & 0 \\
0 & -1 & 0 \\
0 & 0 & 0
\end{array}\right) .
$$

$S U(3)$ is broken to $S U(2) \times U(1)$ or $U(1) \times U(1)$ respectively. Note that for $S U(N)$ with fundamental matter only the pattern of symmetry breaking is fixed because an $S U(N)$ transformation can bring fundamentals to a standard form. However, for exceptional groups a unique standard form for the fundamentals does not exist. Even if we had a concrete representation of the exceptional groups (for example $26 \times 26$ matrices for $F_{4}$ ) it would still be difficult to determine all the breaking patterns. Therefore we exploit a method based on roots and weights. The advantage is that starting from a Dynkin diagram one can determine the roots and weights in a finite number of steps 15, 16, and then our analysis uses an additional finite number of steps. Therefore our method is programmable. 
Therefore, to analyze the patterns of symmetry breaking we abandon tensor notation for the Higgs fields and return to the more basic representation in terms of weights. Recall that generators $\left(E_{\alpha}\right)$ are labeled by roots $(\alpha)$ and act on weights $(\mu)$ like raising and lowering operators $\left(E_{\alpha}\right.$ and $\left.E_{\alpha}^{\dagger}=E_{-\alpha}\right)$. The idea is to give a vacuum expectation value (VEV) directly to a weight $\mu_{i}$ or to a linear combination of some weights. To see how the generators act on the Higgs corresponding to the weight $\mu_{i}$ we just determine how the roots act on the weight $\mu_{i}$. By construction the action of roots on weights is restricted to motions within the weight lattice of the corresponding representation. Either the root moves the weight to some other weight or annihilates it.

It would seem that the solution to our problem is at hand, because given a weight with a VEV the roots that do not annihilate it correspond to broken generators. A slightly modified version of this statement and its converse that unbroken generators correspond to roots that annihilate the weight are true. There are two subtleties:

- The operators $E_{ \pm \alpha}$ are not hermitian. The pattern of symmetry breaking is determined from the hermitian generators constructed from them, $T_{+}=E_{\alpha}+E_{-\alpha}$ and $T_{-}=i\left(E_{\alpha}-E_{-\alpha}\right)$. If at least one of the two operators $E_{ \pm \alpha}$ is broken then both $T_{+}$and $T_{-}$are broken.

- If two roots move the weight $\mu_{i}$ with a VEV to the same weight $\mu_{j}$ then a linear combination of roots remains unbroken. This occurs when the roots are at the origin, ie, when the generators are in the Cartan subalgebra. It also may happen if the $\mathrm{VEV}$ is given to a linear combination of weights.

In sum, given a VEV to a weight $\mu_{i}$ and a root $\alpha$ that moves the weight to $\mu_{j} \neq \mu_{i}$, then both $E_{\alpha}$ and $E_{-\alpha}$ are broken generators. If there are $n$ elements of the Cartan subalgebra then one can always find $n-1$ unbroken linear combinations. If a $\mathrm{VEV}$ is given to a linear combination of weights and $n$ roots map this combination to the same weight, then only $n-1$ of the corresponding generators are unbroken."

While the considerations above apply to any field theory, for SUSY theories there are further simplifications. If the gauge symmetry of a SUSY

\footnotetext{
${ }^{1}$ Determining these combinations can prove difficult. Acting with roots on weights is only defined up to phases. The only requirement on these phases is that the whole algebra
} 
theory is $G$, then the potential is in fact invariant under a larger symmetry, namely the complexified group $G_{c}$. If the symmetry is spontaneously broken $G \rightarrow H$, then the potential of the low energy theory has $H_{c}$ symmetry. Therefore, in a supersymmetric vacuum both lowering and raising operators must be broken simultaneously.2

Let us return to our illustrative example, $S U(3)$ with adjoint matter. The root diagram is

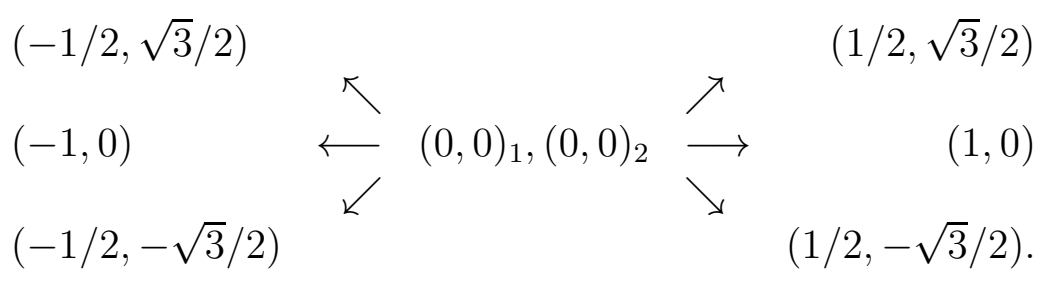

In this example the weight diagram of the matter field is exactly the same.

Given, for example, a VEV to $|(1 / 2, \sqrt{3} / 2)\rangle$ the roots that annihilate it are

$$
\left((0,0)_{1}-1 / \sqrt{3}(0,0)_{2}\right),(1,0),(1 / 2, \sqrt{3} / 2),(-1 / 2, \sqrt{3} / 2),
$$

but only the first gives an unbroken generator, because the last three have hermitian conjugates which are broken. The symmetry is broken down to $U(1)$. The alert reader may be concerned about adjoint breaking to a nonmaximal subgroup. In this example we have given a VEV to a complex weight, which cannot be accomplished with a single hermitian matrix of scalars $A$. However, two scalar fields $A$ and $B$ will accomplish this, and it is clear that unaligned VEVs to two adjoints can break $S U(3)$ to $U(1)$. It is also clear that this vacuum is not supersymmetric. This follows from the general considerations above, but one can easily check that the D-flatness condition is not satisfied.

be consistent. If, for example, two products of simple roots move from $\mu_{i}$ to $\mu_{j}$

$$
E_{\alpha_{1}} E_{\alpha_{2}} \cdots E_{\alpha_{n}} \mu_{i}=e^{i \phi_{1}} \mu_{j} \text { and } E_{\beta_{1}} E_{\beta_{2}} \cdots E_{\beta_{n}} \mu_{i}=e^{i \phi_{2}} \mu_{j} .
$$

the phases $\phi_{1}$ and $\phi_{2}$ are not necessarily equal. There are general ways how to determine the phases. But for the following examples we do so on a case by case basis.

${ }^{2}$ However, the reverse is not true: even if both raising and lowering operators are broken simultaneously the vacuum may not be supersymmetric. One must in addition verify that the $D$-flatness conditions are satisfied. 
If we give a VEV to the real combination $|(1 / 2, \sqrt{3} / 2)\rangle+|(-1 / 2,-\sqrt{3} / 2)\rangle$ the unbroken operators are

$$
(0,0)_{1}-1 / \sqrt{3}(0,0)_{2},(1 / 2, \sqrt{3} / 2)+(-1 / 2,-\sqrt{3} / 2) .
$$

The pattern of symmetry breaking is $S U(3) \rightarrow U(1) \times U(1)$. By symmetry, giving a VEV to any other non zero root results in the same breaking pattern.

Consider a VEV of the zero roots. For $\left|(0,0)_{1}\right\rangle$ the unbroken operators are

$$
(0,0)_{1},(0,0)_{2}
$$

and for $\left|(0,0)_{2}\right\rangle$ they are

$$
(0,0)_{1},(-1,0),(1,0),(0,0)_{2}
$$

The first set corresponds to $U(1) \times U(1)$ the second to $S U(2) \times U(1)$.

In the following sections we give further examples illustrating the method with exceptional groups. Although not presented here, we have used this method to check the symmetry breaking patterns of SO-groups with spinors.

\section{A.1.1 $\quad F_{4}$ with}

The 52 roots of $F_{4}$ are

$$
\begin{array}{lrrl} 
\pm e_{i} \pm e_{j} & (24) & \text { with } i \neq j \text { and } i, j=1,2,3,4 \\
\pm e_{i} & (8) & \text { with } i=1,2,3,4 \\
1 / 2\left( \pm e_{1} \pm e_{2} \pm e_{3} \pm e_{4}\right) & (16) & & \\
(0,0,0,0)_{i} & (4) & \text { with } i=1,2,3,4
\end{array}
$$

and the 26 weights of the fundamental representation are

$$
\begin{aligned}
& \pm e_{i} \pm e_{j} \quad(24) \quad \text { with } i \neq j \text { and } i, j=1,2,3,4 \\
& (0,0,0,0)_{a} \quad(2) \text { with } a=1,2 \text {. }
\end{aligned}
$$

Here $\left(e_{i}\right)_{j}=\delta_{i j}$ is the standard 4-dimensional basis of orthonormal vectors, and the numbers in parenthesis denote the number of such roots and weights. Giving a VEV to $\left(\left|\left(e_{1}+e_{2}\right)\right\rangle+\left|-\left(e_{1}+e_{2}\right)\right\rangle\right)$ breaks the gauge group down to $S O(8)$. But if we give the VEV to the zeros we obtain $S O(8)$ or $S O(9)$. 


\section{A.1.2 $E_{6}$ with $\square+\bar{\square}$}

The 78 roots of $E_{6}$ are

$$
\begin{array}{lrl} 
\pm e_{i} \pm e_{j} & (40) & \text { with } i \neq j \text { and } i, j=1,2,3,4,5 \\
1 / 2\left( \pm e_{1} \pm e_{2} \pm e_{3} \pm e_{4} \pm e_{5} \pm \sqrt{3} e_{6}\right) & (32) \text { with even number of minus signs } \\
(0,0,0,0)_{i} & (6) \text { with } i=1,2,3,4,5,6 .
\end{array}
$$

The 27 weights of the fundamental representation are

$$
\begin{aligned}
& 2 / \sqrt{3} e_{6} \\
& \pm e_{i}-1 / \sqrt{3} e_{6} \\
& 1 / 2\left( \pm e_{1} \pm e_{2} \pm e_{3} \pm e_{4} \pm e_{5}+1 / \sqrt{3} e_{6}\right) \\
& \text { (10) with } i=1,2,3,4,5 \\
& \text { (16) with odd number of minus signs. }
\end{aligned}
$$

The weights of the antifundamental representation are the negative of the weights of the fundamental representation.

Now give a VEV to $\left|\left(2 / \sqrt{3} e_{6}\right)\right\rangle$. The roots that annihilate it are

$$
\pm e_{i} \pm e_{j}, 5 \text { zero roots and } 1 / 2\left( \pm e_{1} \pm e_{2} \pm e_{3} \pm e_{4} \pm e_{5}-\sqrt{3} e_{6}\right) \text {. }
$$

The last 16 do not give unbroken generators. The unbroken roots precisely correspond to the roots of $S O(10)$. In a SUSY theory this pattern of symmetry breaking requires in addition an antifundamental VEV to $\left|\left(-2 / \sqrt{3} e_{6}\right)\right\rangle$ (for D-flatness).

There are alternative breaking patterns. If one gives a VEV to $\left|\left(2 / \sqrt{3} e_{6}\right)\right\rangle+$ $\left|\left(e_{5}-1 / \sqrt{3} e_{6}\right)\right\rangle+\left|\left(-e_{5}-1 / \sqrt{3} e_{6}\right)\right\rangle$ then $E_{6}$ breaks to $F_{4}$.

\section{A.1.3 $E_{7}$ with}

The 133 roots are

$$
\begin{aligned}
& \pm e_{i} \pm e_{j} \\
& 1 / 2\left( \pm e_{1} \pm e_{2} \pm e_{3} \pm e_{4} \pm e_{5} \pm e_{6} \pm \sqrt{2} e_{7}\right) \\
& \pm \sqrt{2} e_{7} \\
& (0,0,0,0)_{i}
\end{aligned}
$$

(60) with $i \neq j$ and $i, j=1,2,3,4,5,6$

(64) with odd number of minus signs for $e_{1}$ to $e_{6}$

(7) with $i=1,2,3,4,5,6,7$

and the 56 weights of the fundamental representation are

$$
\begin{aligned}
& \pm e_{i} \pm 1 / \sqrt{2} e_{7} \\
& \text { (24) with } i=1,2,3,4,5,6 \\
& 1 / 2\left( \pm e_{1} \pm e_{2} \pm e_{3} \pm e_{4} \pm e_{5} \pm e_{6}\right) \\
& \text { (32) with even number of minus signs. }
\end{aligned}
$$

Giving a VEV to $\left|1 / 2\left(e_{1}+e_{2}+e_{3}+e_{4}+e_{5}+e_{6}\right)\right\rangle+\left|-1 / 2\left(e_{1}+e_{2}+e_{3}+e_{4}+e_{5}+e_{6}\right)\right\rangle$ breaks $E_{7}$ to $E_{6}$. 


\section{References}

[*] e-mail address: bgrinstein@ucsd.edu

[†] e-mail address: dnolte@ucsd.edu

[1] N. Seiberg, Phys. Rev. D49, 6857 (1994), hep-th/9402044; Nucl. Phys. B435, 129 (1995), hep-th/9411149

[2] K. Intriligator and N. Seiberg, Nucl. Phys. Proc. Suppl. 45BC (1996) 1; N. Seiberg, The Power Of Duality: Exact Results In 4-D SUSY Field Theory, talk given at the Joint Meeting of the International Symposium on Particles, Strings and Cosmology and the 19th Johns Hopkins Workshop on Current Problems in Particle Theory), Baltimore, MD, 22-25 Mar 1995, PASCOS/Hopkins 1995, p. 183-198

[3] C. Csaki, M. Schmaltz and W. Skiba, Phys. Rev. D55, 7840 (1997), hep-th/9612207

[4] B. Grinstein and D. Nolte, Systematic Study Of Theories With Quantum Modified Moduli, preprint UCSD-PTH-97-26, hep-th/9710001

[5] C. Csaki and W. Skiba, preprint LBL-41320, hep-th/9801173

[6] N. Seiberg and E. Witten, Nucl. Phys. B426, 19 (1994)

[7] N. Seiberg and E. Witten, Nucl. Phys. B431, 484 (1994)

[8] P. Pouliot, Phys. Lett. B367, 151 (1996)

[9] P. Pouliot and M.J. Strassler, Phys. Lett. B370, 76 (1996); Phys. Lett. B375, 175 (1996)

[10] E. Poppitz, Y Shadmi and S. Trivedi, Nucl. Phys. B480, 1251996 hep-th/9605113

[11] K. Intriligator , Phys. Lett. B336, 409 (1994) hep-th/9407106

[12] S. Dimopoulos and J. Preskill, Nucl. Phys. B199, 206 (1982)

[13] P. Cho, Phys. Rev. D56, 5260 (1997), hep-th/9702059 
[14] P. Cho, Moduli In Exceptional SUSY Gauge Theories, preprint HUTP97-A099, hep-th/9712116

[15] J.E. Humphreys, Introduction to Lie Algebras and Representation Theory, Springer (1972)

[16] H. Georgi, Lie Algebras in Particle Physics, Addison-Wesley (1982)

[17] G. Dotti, A.V. Manohar and W. Skiba, Supersymmetric gauge theories with a free algebra of invariants, preprint UCSD/PTH 98-09, hep-th/9803087 